\title{
Mirada ecológica en la construcción del proyecto de vida de jóvenes rurales
}

\author{
Camila Yomara Erazo-Borrás ${ }^{a}$ \\ Universidad de Nariño, Colombia
}

\author{
Ana Karen Ceballos-Mora, Mg. ${ }^{b}$ \\ Universidad de Nariño, Colombia \\ Johana Madelyn Matabanchoy-Salazar ${ }^{c}$ \\ Universidad de Nariño, Colombia
}

camilaerasso@gmail.com

\section{Resumen (analítico)}

El proyecto de vida de la juventud rural colombiana transita por dificultades en su planeación y desarrollo. Por ende, este estudio busca identificar los factores que interfieren en la construcción del proyecto de vida en jóvenes rurales, con una metodología de investigación cualitativa, enfoque etnometodológico y diseño transversal. Se emplearon como técnicas de recolección de información entrevistas semiestructuradas, grupos focales, observación y análisis documental. Participaron 117 adolescentes, ocho docentes y seis padres de familia. Los resultados facilitaron comprender los factores individuales y sociales que inciden en el proyecto de vida de la juventud rural desde el modelo ecológico, que aborda la relación del adolescente rural con las características de su contexto y específicamente desde el microsistema, mesosistema, exosistema y macrosistema.

\section{Palabras clave}

Juventud rural; psicología del desarrollo; dinámica social; deserción escolar; participación comunitaria.

\section{Thesauro}

Tesauro de Ciencias Sociales de la Unesco.

\section{Para citar este artículo}

Erazo-Borrás, C. Y., Ceballos-Mora, A. K., \& Matabanchoy-Salazar, J. M. (2022). Mirada ecológica en la construcción del proyecto de vida de jóvenes rurales. Revista Latinoamericana de Ciencias Sociales, Niñez y Juventud, 20(1), 1-25.

https://dx.doi.org/10.11600/rlcsnj.20.1.5255

\section{Historial}

Recibido: 08.02.2021

Aceptado: 12.07.2021

Publicado: 15.12 .2021

\section{Información artículo}

Este artículo se deriva del macroproyecto Cultivando oportunidades para el crecimiento personal y el desarrollo institucional, formulado desde el Departamento de Psicología de la Universidad de Nariño. La investigación no obtuvo financiación institucional, pero sí hubo apoyo logístico y de gestión por parte del Centro Educativo Municipal El Campanero y la Universidad de Nariño. La información presentada se recolectó entre marzo y junio de 2019. Área: ciencias sociales, psicología. Subárea: interdisciplinaria. 


\section{Ecological perspective of the construction of life projects by rural youth}

\section{Abstract (analytical)}

The life projects of Colombian young people in rural areas are facing difficulties in terms of planning and implementation. This study sought to identify the factors that affect rural young people's construction of life projects. This involved the use of a qualitative research methodology, ethnomethodological approach and cross-sectional design. Semi-structured interviews, focus groups, participant observation and documentary analysis were selected as data collection techniques. A total of 117 adolescents, 8 teachers and 6 parents participated. Using the ecological model, the results facilitated understanding of the individual and social factors that influence the life projects of rural youth. The study identified the importance of the relationship of rural adolescents with the characteristics of their context and specifically the microsystems, mesosystems, exosystems and macrosystems they inhabit.

Keywords

Rural youth, developmental psychology, social dynamics, student dropouts, community participation.

\section{Uma perspectiva ecológica na construção do projeto de vida dos jovens rurais}

Resumo (analítico)

O projeto de vida da juventude rural colombiana está passando por dificuldades em seu planejamento e desenvolvimento, portanto, este estudo procura identificar os fatores que interferem na construção do projeto de vida na juventude rural, com uma metodologia de pesquisa qualitativa, abordagem etnometodológica e desenho transversal. Foram utilizadas entrevistas semiestruturadas, grupos de foco, observação e análise documental como técnicas de coleta de dados. Participaram um total de 117 adolescentes, 8 professores e 6 pais participaram. Os resultados facilitaram uma compreensão dos fatores individuais e sociais que influenciam o projeto de vida dos jovens rurais a partir do modelo ecológico, que aborda a relação dos adolescentes rurais com as características de seu contexto e especificamente do microssistema, mesossistema, exossistema e macrossistema.

\section{Palavras-chave}

Juventude rural, psicologia do desenvolvimento, dinâmica social, abandono escolar, participação comunitária.

Información autoras

[a] Psicóloga, Universidad de Nariño (Colombia). (iD) 0000-0002-4084-2283. H5: 0. Correo electrónico: camilaerasso@gmail.com

[b] Psicóloga, Universidad de Nariño. Magíster en Salud Pública, Universidad de Nariño. Docente del Programa Tecnología en Promoción de la Salud, Universidad de Nariño (Colombia). (iD) 0000-0002-4980-4056. H5: 3. Correo electrónico: ceballosank@udenar.edu.co

[c] Especialista en Seguridad y Salud en el Trabajo, Universidad de Nariño. Psicóloga, Universidad de Nariño. Docente del Programa de Psicología de la Universidad de Nariño (Colombia). (iD) 0000-0002-3831-7252. H5: 1. Correo electrónico: udenarmade7@udenar.edu.co 


\section{Introducción}

no de los pilares fundamentales en la formación del ser humano es la cons-
trucción del proyecto de vida, entendido como un sistema principal del cual parte aquello que se anhela ser o hacer y que integra tanto la personalidad como las áreas vitales del desarrollo (Acevedo et al., 2020; D’Angelo, 200o). En este sentido, el proyecto de vida permite al individuo situarse en la sociedad, interactuar y adquirir roles para desenvolverse en la cotidianidad, convirtiéndose en un proceso flexible y constante, de suma importancia en cada etapa del desarrollo humano, especialmente en la adolescencia (Suárez-Barros et al., 2018); esta última es una etapa en la cual el individuo toma decisiones fundamentales que repercutirán en los ámbitos laboral, sociocultural, familiar, académico y económico (Ayala \& Carvajal, 2016; Barbosa-Martínez et al., 2016; Klein \& Arantes, 2016; Suárez-Barros et al., 2018).

En la configuración del proyecto de vida, siguiendo los ideales y planes para el futuro, las acciones se centrarán principalmente en el logro de objetivos o metas; lo cual, a su vez, produce beneficios en las esferas personal y social. Entre dichos resultados se encuentran la realización personal, el incremento del potencial individual, la adopción de un estilo de vida saludable, la prevención de enfermedades y mayores logros tanto personales como profesionales (D’Angelo, 2000, 2002; Ministerio de Educación [Ecuador], 2018).

D’Angelo (1986) y el Fondo Internacional de Desarrollo Agrícola ([Fida], 2019) plantean que una definición inadecuada de los objetivos vitales puede repercutir negativamente en la conducta y en el proyecto de vida de los adolescentes, lo cual desemboca en confusiones y contradicciones al momento de elegir una profesión y, como consecuencia, en una conducta laboral inestable, insatisfacción, apatía o una indefinición de una orientación estable en el futuro. Así las cosas, se comprende que las decisiones que los jóvenes toman en su cotidianidad se encuentran altamente influenciadas por su proyecto de vida y estas, a su vez, afectan el futuro (Barbosa-Martínez et al., 2016). 
Al hablar en particular de la juventud rural, esta cuenta con características propias ya que forman parte de comunidades pequeñas que están distantes de la ciudad, el comercio y la industria (Pardo, 2017; Rodríguez-Brito, 2019). Estas poblaciones han permanecido en el sector agrícola y, por generaciones, también han sido objeto de desigualdad y exclusión territorial. Sin embargo, la actual generación de jóvenes rurales difieren de sus antecesores al ser potenciales agentes de transformación de sus comunidades y territorios, puesto que poseen mayor flexibilidad, disposición al cambio y apertura a la innovación, teniendo en cuenta su mayor nivel de acceso a la información, a servicios públicos básicos, a la educación preescolar, básica y media, así como el acceso a las tecnologías de la comunicación, en comparación con la población rural de generaciones anteriores. Aun así, la brecha de desigualdad y exclusión social es un fenómeno notable que genera limitaciones para participar en la vida política y económica, tanto de la comunidad como del país (Fida, 2019; Pardo, 2017; Rodríguez-Brito, 2019).

La educación en contextos rurales del territorio colombiano enfrenta diversos retos, y la baja permanencia de los estudiantes es uno de los principales. En 2013 se encontró que el $13.8 \%$ de los jóvenes rurales entre 12 y 15 años de edad no asistieron a la educación secundaria; mientras que el 39.4\% de los jóvenes de 16 y 17 años se encontraban desligados del sistema educativo (Departamento Nacional de Planeación [DNP], 2015). Esto se corrobora años después en el Plan Especial de Educación Rural, en el cual es notable la diferencia de las cifras respecto a la permanencia escolar en Colombia, con un promedio de 6 años en las zonas rurales y de 9.6 en las urbanas en 2016 (Ministerio de Educación Nacional [MEN], 2018).

Comprender cómo se da la construcción de un proyecto de vida en el adolescente rural implica reconocer que este se gestiona multidireccionalmente en diferentes esferas vitales; es decir, son varios sistemas interconectados y cada uno se relaciona de forma recíproca entre sí. En este sentido, aquello que sucede en uno de sus entornos afecta a los demás y, por ende, al adolescente rural. Es así como el proyecto de vida en adolescentes rurales se debe comprender como un fenómeno transversal al individuo y a los entornos que lo rodean.

Según lo expresado por Bronfenbrenner (1987) y Suárez-Barros et al. (2018), entender los ambientes en los cuales el ser humano se desenvuelve es crucial para poder detectar la variedad de factores que influyen en su desarrollo. Bronfenbrenner (1987) plantea el modelo ecológico, el cual aborda al desarrollo humano como una relación constante de un sujeto activo con las características versátiles de su ambiente, entendiendo que ambos influyen en el otro recíprocamente. Este modelo ecológico está compuesto por 
estructuras seriadas y concéntricas, en el que cada una se encuentra contenida dentro de otra, y en el sistema más interno se ubica la persona en desarrollo (Ortega et al., 2021).

Bronfenbrenner (1987) propone cuatro sistemas que forman parte del modelo ecológico: el microsistema, el mesosistema, el exosistema y el macrosistema. El microsistema se refiere a la interacción bilateral entre el individuo y su entorno inmediato; el mesosistema corresponde a la relación con aquellos lugares y personas con los que el sujeto interactúa en su cotidianidad aparte del microsistema; el exosistema es el contexto con el cual el individuo no interactúa directamente, pero que influye en su desarrollo diario y el macrosistema abarca las características culturales, sociales y políticas en las que está inmersa la persona.

Entonces se retoman los cuatro sistemas anteriormente descritos con la finalidad de discernir los factores que intervienen en la relación de la juventud rural y su entorno ecológico frente a la construcción de su proyecto de vida. Estos sistemas permiten comprender cómo la interacción de los entornos repercute en la manera en la que la juventud rural construye valores, creencias, expectativas y decisiones (Schmitt \& Dos Santos, 2013). Teniendo en cuenta lo anterior, es importante entender cuáles son los factores de cada uno de los sistemas del modelo ecológico que interfieren en la construcción del proyecto de vida en jóvenes rurales.

Actualmente hay poca información respecto al proyecto de vida en la población rural. Si bien existen diversos estudios acerca de los diferentes componentes del proyecto de vida en población joven, es notable la ausencia de información sobre aquellos factores que se relacionan con la construcción del proyecto de vida en contextos rurales. Por lo tanto, el objetivo general de esta investigación se orientó a identificar los que interfieren en la construcción del proyecto de vida en jóvenes rurales desde la perspectiva del modelo ecológico.

\section{Metodología}

El presente estudio fue de tipo cualitativo con enfoque etnometodológico y diseño transversal, lo cual permitió abordar las creencias sobre las dinámicas culturales por medio de un acercamiento a profundidad de lo que acontece en torno al proyecto de vida de los jóvenes rurales; lo anterior, a partir de las percepciones y experiencias que comparten ellos mismos, sus padres y sus educadores, considerando también las dinámicas 
sociales (Jiménez-Ruiz \& Almansa, 2017; Ocádiz, 2016). Además, se respetaron las explicaciones dadas por quienes participaron y los términos que utilizaron.

Se contó con la participación de 117 jóvenes pertenecientes a educación básica secundaria del Centro Educativo El Campanero, ubicado a $14.7 \mathrm{~km}$ de la cabecera municipal de San Juan de Pasto, en Colombia. Las edades de los participantes oscilan entre los 11 y 16 años, de los cuales el $59.8 \%$ son hombres y el $40.17 \%$ mujeres. Además, participaron ocho $(38 \%)$ de los 21 docentes del centro educativo y seis padres de familia.

En la selección de los participantes se tuvo como criterios de inclusión: que fueran estudiantes del centro educativo, entre sexto a noveno grado, que contaran con el consentimiento informado de sus padres o tutores y que deseasen participar voluntariamente. En el caso de los docentes, se consideró relevante que tuvieran una amplia experiencia como docentes rurales - en específico en el centro educativo-y que quisieran tomar parte de forma voluntaria. Por último, los padres de familia fueron invitados a participar con la estrategia bola de nieve y se identificó a tres de ellos como líderes de la comunidad, quienes, a su vez, motivaron a otros a participar en el estudio.

La recolección de información se realizó mediante entrevistas semiestructuradas, grupos focales, observación y análisis documental, los cuales facilitaron la comprensión y el entendimiento intercultural a partir de la comunicación empática (Jiménez-Ruiz \& Almansa, 2017).

Se efectuaron 14 entrevistas semiestructuradas: seis a padres de familia y ocho a docentes de la institución. Para ambos grupos se abordaron las temáticas de resignificación de proyecto de vida, resiliencia, toma de decisiones, resolución de conflictos, deserción estudiantil y bajo desempeño académico. La entrevista a padres de familia contó con 16 preguntas y la entrevista a docentes con 15, las cuales fueron validadas por expertos. Esta técnica cualitativa parte de la formulación de preguntas preestablecidas y flexibles (Díaz-Bravo et al., 2013; Hernández et al., 2014; Lopezosa, 2020).

Asimismo, se desarrollaron cinco grupos focales con estudiantes acerca de las fortalezas y los aspectos de mejora en los planes para el futuro en los aspectos familiar, profesional y académico, así como de resolución de conflictos, toma de decisiones, percepciones sobre los causales de deserción estudiantil y bajo desempeño académico. Igualmente, se diseñaron 23 preguntas para los grupos focales, las cuales también fueron revisadas por expertos.

Además, se indagó sobre las creencias y los prejuicios que los estudiantes tienen en cuanto a la vida laboral y académica en contextos rurales y urbanos, y en relación con su 
proyecto de vida según su entorno. Lo anterior estuvo enfocado a orientar la construcción del proyecto mencionado, en función de los diferentes sistemas y la transformación de los prejuicios sobre la vida laboral o académica. Se trabajó mediante preguntas guías, realizadas durante la interacción entre los participantes y el investigador, lo cual suscitó creencias y experiencias en un corto tiempo (Hamui \& Varela, 2013; Hernández et al., 2014; Rodas \& Pacheco, 2020). A lo largo de la investigación se efectuó la observación para describir, analizar y argumentar la realidad visualizada mediante una perspectiva científica (Campos \& Martínez, 2012; Schettini \& Cortazzo, 2016).

El macrosistema fue abordado desde el análisis documental, el cual permitió analizar el contenido de políticas de educación en ruralidad y documentos institucionales, con la finalidad de tener mayor comprensión sobre la implementación de las políticas públicas para la educación rural en el país y que aporten al proyecto de vida de jóvenes rurales.

\section{Plan de análisis de datos}

En la sistematización y el análisis de información, se utilizó el software ATLAS.ti (v. 7.5.4) y fueron desarrolladas las siguientes etapas: 1) transcripción de entrevistas semiestructuradas, grupos focales y observación; 2) importación de información, la cual fue cargada para su análisis al software ATLAS.ti; 3 ) lectura exhaustiva de las repeticiones y concordancias entre los discursos; 4) codificación de datos para la identificación de categorías deductivas y emergentes; de esta manera, una vez que se llegó a una saturación de los datos, se organizaron las categorías con relación al modelo ecológico; y 5) triangulación de información. Las categorías de la investigación se presentan en la tabla 1.

\section{Tabla 1}

Categorías y subcategorías derivadas del análisis de datos

\begin{tabular}{|c|c|c|}
\hline Categoría & Subcategoría & Tipo \\
\hline \multirow{3}{*}{ Microsistema } & Apoyo familiar & Deductiva \\
\hline & Autoestima & Deductiva \\
\hline & Motivación & Emergente \\
\hline \multirow{3}{*}{ Mesosistema } & Apoyo social & Deductiva \\
\hline & Centro educativo & Deductiva \\
\hline & Sentido de comunidad & Emergente \\
\hline Exosistema & Características socioculturales & Deductiva \\
\hline \multirow{2}{*}{ Macrosistema } & Políticas públicas & Deductiva \\
\hline & Entidades del gobierno & Deductiva \\
\hline
\end{tabular}


Esta investigación se desarrolló con los lineamientos éticos estipulados en el Código Deontológico y Bioético de la Ley 1090 de Colombia (Congreso de la República, 2006), por el cual se regula el ejercicio de los profesionales de psicología. Se contó con el aval de la institución y en todo momento se salvaguardaron los principios tanto de confidencialidad y autonomía como de beneficencia en el proceso investigativo; para ello se garantizó el diligenciamiento del consentimiento informado por parte de los docentes y padres de familia, así como también el consentimiento asistido de los estudiantes.

\section{Resultados}

El presente estudio se desarrolló en el Centro Educativo Municipal El Campanero, el cual está conformado por cinco sedes en las veredas El Campanero, Bellavista, San Antonio de Casanare, San José de Casanare y Alto Casanare, del corregimiento de Catambuco, municipio de Pasto. La población de este territorio tiene como principal medio productivo la agricultura y la ganadería, caracterizándose por el arraigo cultural a sus costumbres y tradiciones, situación que ha incidido significativamente en la construcción del proyecto de vida de los jóvenes.

De esta manera, se consideró prioritario identificar los factores que interfieren en la construcción del proyecto de vida en jóvenes rurales desde la perspectiva del modelo ecológico. A partir de la teoría ecológica de Bronfenbrenner $(1987,2005)$ fue posible identificar los factores que desde cada sistema se relacionan con la construcción del proyecto de vida (figura 1).

\section{Microsistema}

Aquí se retomó al microsistema como aquel entorno inmediato en el cual está inmerso el o la adolescente rural y con el cual se relaciona directamente, al ser parte de su cotidianidad e influir significativamente en su realidad; en este punto también se encuentra el sujeto y sus características personales. En específico, se identificó que las categorías deductivas autoestima y apoyo familiar, así como la categoría emergente motivación interfieren en la construcción del proyecto de vida. 
Figura 1

Factores que interfieren en la construcción del proyecto de vida de los jóvenes rurales

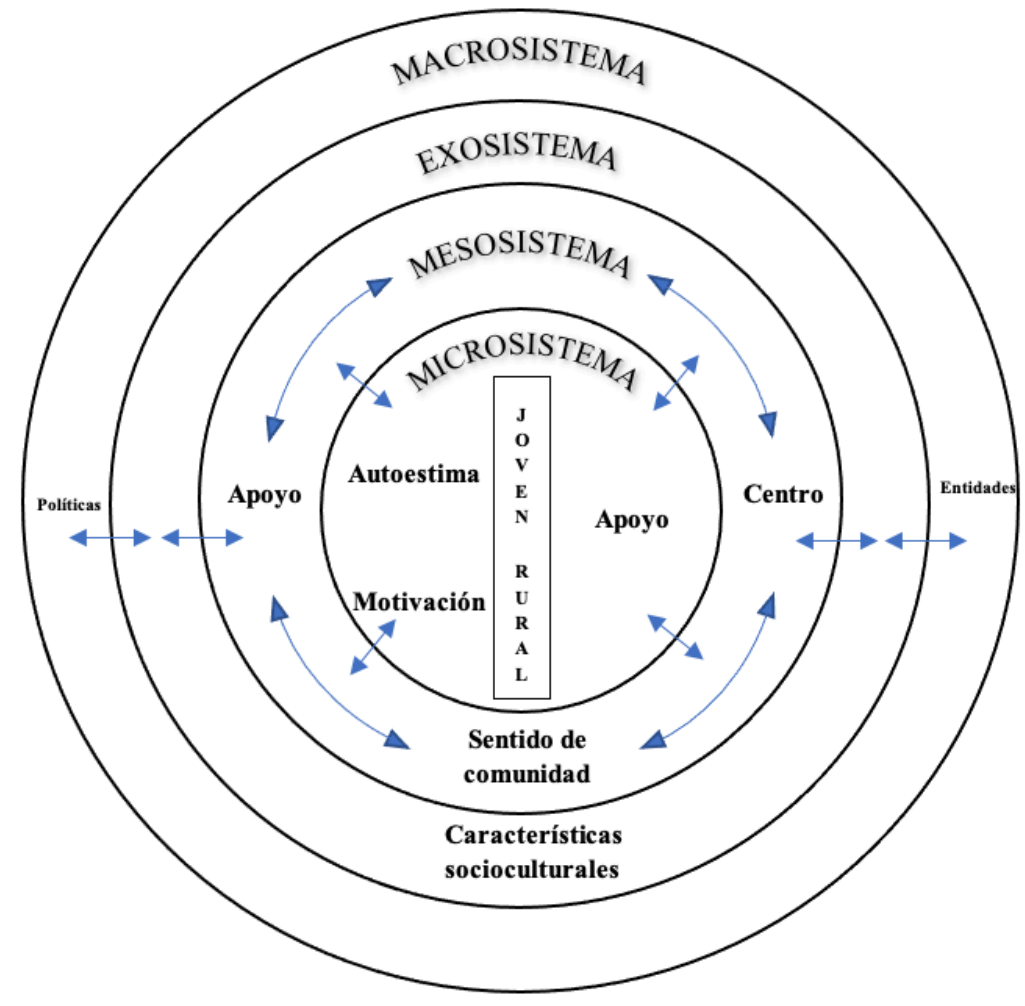

Al analizar la información obtenida con respecto a la autoestima, se evidenció que los participantes mostraron incertidumbre sobre sus propias fortalezas, debilidades, anhelos y afinidades. Ligado a ello, también fue posible entrever que un cierto número de jóvenes dudan sobre su autoeficacia y capacidad de alcanzar un proyecto de vida, tanto por dentro como por fuera de la vida rural, así como muestran aspectos de mejora en cuanto al desarrollo de su autoestima.

$\mathrm{Al}$ respecto, una docente manifiesta: «Pueden llegar a ser mejores, mejor como persona; enseñarles cómo bañarse, en su aseso personal. Que se sientan ellos bien, su autoestima, desde esa parte hasta la parte profesional» (SJ11). Otro docente expone: «Acá se citan más que todo por el aseo personal, cuando faltan mucho» (S/7).

Esto indica que en la autoestima de la juventud rural hay varios aspectos que se deben fortalecer, entre ellos, el autocuidado. Se pudo evidenciar que la mayoría de la población trata como un tabú el autocuidado del cuerpo y el manejo de la sexualidad. Por 
ende, descuidar la presentación personal y desconocer la importancia de cuidar su cuerpo representan un factor de riesgo respecto al desarrollo óptimo de su autoestima.

En cuanto al apoyo familiar, se identificó que las relaciones con sus familiares más cercanos son significativamente importantes, así como el acompañamiento parental afectivo, el cual con frecuencia está articulado al proceso académico del estudiante. A partir de las entrevistas a padres de familia fue posible observar que el apoyo afectivo hacia los hijos se caracteriza por expresiones como abrazos y palabras de cercanía. En este sentido, los padres de familia mencionan:

Cuando hace mal las cosas, uno le explica y cuando lo hace bien, uno le da un abrazo. (SJ6) Cuando está bien, ya ve mi'ja..., un abrazo, así está bien, así me gusta. Bueno, hijo, un abrazo, así sí aporto a la enseñanza. Si sigue así, todos los días le doy abrazo. ( $\left.\mathrm{SJ}_{3}\right)$

No obstante, se evidencia la importancia de articular este apoyo afectivo con un acompañamiento parental más activo frente al desempeño académico de los adolescentes, al considerar que se presenta un bajo seguimiento del proceso educativo por parte de algunos padres de familia. En este punto, según la información arrojada, se revelan en el aspecto familiar dos factores de riesgo de bajo rendimiento académico y deserción escolar que afectan al proyecto de vida.

El primero se relaciona con que una parte de los padres de familia priorizan el tiempo que el adolescente dedica al trabajo en labores del campo por encima al que se dedica a la formación educativa. Entonces algunos padres tienen poco interés para que los jóvenes continúen sus estudios después de terminar la primaria. Al respecto, una docente refiere:

Por el contexto, acá los papitos no le muestran mucho interés de que ellos sigan su estudio. Es como esa forma en que ellos ven, ven como que no, el campo es mejor; que mejor es cultivar, sembrar. Entonces se impulsan en eso, como aprender a leer y a escribir y seguir sus trabajos del campo. (SJ15)

El segundo factor es una baja confianza por parte de los padres de familia sobre las capacidades de los jóvenes, lo cual limita la toma de decisiones con base en su propio criterio:

Uno siempre trata en reuniones de padres de familia cuando ya se los tiene aquí; tratar de hablarles de cómo ayudarles a sus hijos, de cómo estar pendiente de ellos, de que no les corten las alas, de que si ellos quieren estudiar, no digan no hasta tal grado y ya no más. 
Entonces hasta los padres también hay que hablarles para que ellos también crean en sus hijos, porque falta. Ellos no creen en las capacidades que puedan tener para llegar a algo más. $(\mathrm{S} J 15)$

Desde otra perspectiva, en las entrevistas semiestructuradas y grupos focales se encontró que algunos padres de familia rurales han contemplado que sus hijos se desempeñen en ocupaciones laborales distintas a las de la ruralidad o que lleven a cabo estudios para desarrollarse profesionalmente, ya sea en el campo u otro sector, mostrando disposición para apoyarlos económica y afectivamente. Así lo evidencia esta madre de familia: «Yo quiero como decir que tengan un trabajo que no sea como nosotros; que sea, cómo le digo, que no sea en el campo porque el campo es duro, que sea en la ciudad» $\left(\mathrm{SJ}_{3}\right)$.

Los hallazgos indicaron que el apoyo familiar es indispensable y repercute en cómo el y la joven rural construyen su proyecto de vida, por la carga socioafectiva que representa para ellos. Incluso encontramos en repetidas ocasiones que, al abordar esta temática, los estudiantes contemplan a su familia como las primeras personas a quienes beneficiarían directamente mediante la elaboración de su proyecto de vida.

Respecto a la motivación, se observa que es poco el interés de los jóvenes para permanecer en la escolaridad y construir su proyecto de vida. Los docentes refieren que muchos de los jóvenes prefieren iniciar y mantener una vida laboral agrícola a edad temprana, dado que con ello dan lugar a la generación de ingresos económicos; por otro lado, está la falta de motivación intrínseca de los estudiantes para permanecer en un proceso educativo a largo plazo, además del bajo acompañamiento parental frente a su formación académica. Así lo refieren a continuación los docentes entrevistados: «Ellos piensan diferente, en formar una familia en tan temprana edad; ellos no quieren..., quieren trabajar, quieren tener plata. Solo piensan en eso, y uno qué puede hacer» (SJ15). «Una es la motivación de los niños, de pronto las dificultades que el niño encuentra para aprender, no entiendo, esto no me sirve para nada» (SJ11). «Uno por el trabajo, por la situación familiar y porque no se sienten motivados, no le hallan sentido a estudiar» (SJ11).

Los estudiantes resaltaban dos aspectos notables en cuanto a la baja motivación para continuar en un proceso educativo y configurar un proyecto vital; por una parte, la preferencia por apoyar labores agrícolas con la finalidad de obtener dinero y, por otra, no encontrarle sentido o gusto a mantenerse dentro de un proceso académico. Cabe resaltar que el primer criterio fue referenciado, en su gran mayoría, por adolescentes entre octavo y 
noveno grado. De forma similar, los padres de familia refirieron ambas causas asociadas a la deserción del estudiante rural.

También fue posible identificar mediante dibujos, relatos y frases que, al abordar la temática de proyecto de vida, los jóvenes rurales, a modo reflexivo, comprendían que en este proceso podía haber obstáculos que debían afrontar, e igualmente lograban encontrarle sentido a permanecer en un proceso educativo a partir de la reflexión crítica en aras de mantener su motivación frente a este.

\section{Mesosistema}

En él se ubican aquellos entornos en los que está inmerso el adolescente rural, correspondiendo a los lugares y las personas con los que se relaciona activamente por fuera de su entorno inmediato (microsistema) y con los que se desenvuelve en su vida cotidiana. Para este caso, se identificaron las categorías deductivas apoyo social y centro educativo, así como la categoría emergente sentido de comunidad, las cuales están relacionadas con la construcción del proyecto de vida.

Respecto al apoyo social, se encontró que la relación entre pares que maneja la juventud rural se caracteriza por ser estrecha, colaborativa y solidaria; asimismo, se evidenció la presencia de imitación de conductas tanto de forma interna (en el aula) como por fuera de la institución. Sin embargo, los adolescentes rurales se diferencian en que siguen los patrones de comportamiento ligados a involucrarse plenamente a una vida laboral agrícola temprana para la obtención de recursos que, de manera paulatina, llevan a la deserción escolar. Aun así, es importante resaltar que las relaciones familiares son más relevantes e influyentes que las establecidas con sus pares frente a las decisiones que toman a corto, mediano y largo plazo. De esta forma lo expresó una docente:

Se ve mucha colaboración también. A veces puede ser negativa porque uno hace la tarea y lo hace copiar al otro. Yo creo que esas cositas también hay que mejorarlas un poco. Creo que, en general, son muy amigos, muy sociables entre ellos, se llevan bien. (SJ13)

En el mesosistema, y particularmente en el centro educativo que sirve a una comunidad rural, los docentes muestran una constante adaptación profesional, didáctica y afectiva a las necesidades de los estudiantes. En contraste con la educación urbana, los docentes reconocen las diferencias entre ambas y las posibles dificultades presentes en el contexto y adoptan una postura flexible ante ellas, teniendo la disposición de crear o 
modificar metodologías de enseñanza a favor de motivar a los estudiantes rurales por permanecer aprendiendo. Incluso es frecuente observar que ellos exceden las funciones de enseñanza para aportar desde su parte humana y personal a los estudiantes y su comunidad.

Desde la perspectiva del personal docente, sus funciones, además de involucrar la enseñanza de conocimiento, trascienden a la formación de habilidades y capacidades que aporten en la vida cotidiana de los jóvenes; de tal manera que se propende por una formación integral como seres humanos. También se evidencia la relevancia de que estos procesos de desarrollo integral y seguimiento a la deserción escolar en contextos rurales estén apoyados por una persona con formación profesional en psicoorientación, dado que esto permite reconocer de forma rápida y oportuna los desencadenantes que afecten psicosocialmente a los adolescentes rurales respecto a su proyecto de vida.

Según la mirada institucional, mediante las entrevistas realizadas a la psicoorientadora, se identificaron alianzas estratégicas establecidas por la institución educativa con diferentes entidades, con las cuales se han implementado programas para promover el desarrollo de habilidades para la vida, prevención del consumo de sustancias psicoactivas, fortalecimiento de las habilidades artísticas y deportivas y participación comunitaria de los jóvenes rurales.

En la categoría emergente de sentido de comunidad, los hallazgos indican que al realizar un abordaje reflexivo sobre esta temática, gran parte de los jóvenes rurales consideran importante, para la construcción autónoma de un proyecto de vida, partir de su sentido de comunidad respetando sus costumbres y tradiciones, pero pensando en su desarrollo y en las necesidades de su contexto. Es así como los estudiantes rurales se distinguen por darle prioridad a la calidad de vida que pueden generar a su comunidad y a su círculo familiar a partir del propio crecimiento personal. En ese sentido, una estudiante puntualizó:

Pensar acerca del proyecto de vida es muy bueno porque me ayuda a pensar y nos impulsa para que en el futuro seamos grandes profesionales. Trabajar en el proyecto de vida a mí me pareció muy bonito porque nos ayuda a reflexionar y muchas veces es pensar ir lejos y así no solo poder ayudar a mi familia sino a otras personas que también lo necesitan. (SJ26)

Otro aspecto por resaltar y relacionado con el sentido de comunidad es el empoderamiento de los jóvenes rurales, el cual ha ido en auge y los reconoce como agentes con autoeficacia, capaces y responsables de transformar su comunidad y territorio al gestionar 
su proyecto de vida. De igual forma, esto se articula al hecho de que los integrantes de la comunidad han desarrollado sentido de pertenencia hacia ella, por lo que se perciben a sí mismos como miembros valiosos de su colectividad para el alcance de las necesidades afectivas, grupales, materiales o psicológicas que se presenten y construyen su propia identidad a partir de su sentimiento de pertenencia con esta.

La búsqueda y formación de líderes comunitarios es un método común y efectivo que esta comunidad rural ejerce para facilitar la comunicación y gestión de recursos entre veredas o con actores externos, y los percibe como personas que representan sus intereses y que actúan a favor del bienestar comunal. Esto se mantiene en algunos jóvenes rurales que desempeñan su liderazgo para fortalecer la comunicación entre los estudiantes de las diferentes escuelas veredales, los docentes y los padres de familia. Así, se convierten en agentes comunitarios dado que representan a la comunidad rural, a los estudiantes de su sede educativa y al centro educativo.

\section{Exosistema}

En este se contemplan aquellos subsistemas en que los jóvenes rurales no participan activamente, pero que afectan su comportamiento; por lo tanto, se identificó que las características socioculturales tienen un papel importante en la construcción del proyecto de vida.

Respecto a dichas características socioculturales en las que están inmersos los jóvenes rurales, se encontró que la mayoría de los habitantes de esta población se movilizan en un territorio claramente delimitado y se relacionan en especial con su propia comunidad, por lo que cada miembro sigue un proceso cíclico de interacción y modo de vivir con su propia comunidad y las veredas próximas que conforman el territorio. De forma similar, fue posible entrever una significativa unión comunal como particularidad coincidente entre los pobladores, porque fue usual encontrar una importante gestión, participación y organización comunitaria. Así, es la propia comunidad la que genera o diligencia cooperativamente los recursos necesarios para cada situación que emerge, asignando roles y funciones en juntas de acción comunal, en eventos como la organización de campeonatos deportivos o celebraciones festivas, o para la recolección de recursos monetarios o materiales.

Lo anterior es un aspecto que fortalece redes de apoyo y se establece como un factor protector que afianza los lazos, el sentido de comunidad y la cooperatividad entre cada actor comunitario. Ello se puede evidenciar en lo manifestado por una docente del plantel: 
Pues aquí de pronto por el mismo entorno son muy allegados todos; no falta que el uno sea primo del otro. Entonces aquí todos se conocen entre todos y, si no, se van conociendo en el camino; cada vez se vuelven más cercanos, se vuelven más amigos. Entonces yo creo que hay como mucha confianza entre ellos y se ve mucha colaboración también. (SJ13)

Por otro lado, esta delimitación dirige la mirada de los jóvenes a un panorama limitado y dificulta reconocer alternativas diferentes a lo culturalmente establecido al momento de pensar en construir un proyecto de vida o tomar decisiones respecto a este. De igual forma, fueron identificados rasgos de personalidad usuales que tienden a la introversión y que son compartidos entre los miembros de esta comunidad, así como lo expresó una docente:

Y en realidad es mostrarles otro mundo, porque ellos en realidad de vez en cuando bajan a Pasto, a tener ese contacto allá. Con ellos de pronto podemos hacer actividades de salidas, que vayan a cine. Entonces como mostrarles otras cosas para que ellos se interesen, porque su contexto les hace ser así introvertidos, calladitos; $y$, por ejemplo, es de la escuela a la casa, de la casa a la escuela, pero por lo general no salen. La vida de ellos es si no están en la escuela se van a trabajar. Entonces es como duro porque para ellos el qué paseo es irse a Las Lajas. (SJ11)

En relación con el fenómeno de deserción escolar, se halló que tanto hombres como mujeres abandonan sus estudios sin terminar la secundaria; sin embargo, se identificó roles desigualitarios muy marcados de género. Por lo general, a las mujeres se las designa para el cuidado del hogar y los hijos, mientras que los hombres se encargan del trabajo de fuerza en labores agrícolas como peones o jornaleros. Ello demuestra un comportamiento y una socialización machistas, que son un factor de riesgo para la deserción, particularmente en las mujeres, como bien lo afirmó una docente:

Otro problema con la deserción, sobre todo con las chicas, [es] un embarazo a temprana edad. Se vuelven mamás rapidito y eso hace que ellas no piensen en otras cosas en un futuro, en una carrera, sino bueno, entonces soy mamá y cuidar a mis hijos, la cocina y de ahí no salen. $\left(S J_{13}\right)$

\section{Macrosistema}

En este se contempló el funcionamiento de las políticas públicas. Desde la posición de Herrera y Rivera (2020), así como de Pardo (2017), los programas de políticas públicas para la educación en Colombia están dirigidos principalmente hacia los jóvenes urbanos, 
mientras aquellos programas para jóvenes rurales están diseñados considerando las condiciones de las ciudades sin estar adaptados a la población objetivo. En este sentido, la falta de políticas públicas se ve remarcada en zonas rurales aisladas de los municipios donde los jóvenes tienen educación básica y primaria en escuelas veredales, que cuentan con menor infraestructura física, personal docente limitado y menor calidad en comparación con las instituciones del casco urbano. Lo anterior se ve reflejado en el hecho de que estudiantes de diferentes grados toman clases en un mismo salón o que un solo docente trabaja con distintos grados e imparte todas las materias.

En la actualidad no hay programas que apoyen la permanencia escolar ni la construcción de su proyecto de vida en zonas rurales mediante programas y recursos estatales, que le permitan a la juventud rural transitar hacia la educación media profesional cuando la institución educativa no cuenta con los recursos para dar acceso a ella (Velasco, 2020). Tampoco hay iniciativas que faciliten a los jóvenes rurales entrar a la educación superior a través de la articulación de la educación media a la técnica en la misma institución (Pardo, 2017). Por otro lado, los únicos proyectos estatales que se encontraban en funcionamiento en la institución educativa objeto de esta investigación fueron aquellos que están implementados en todas las instituciones educativas del país: el Programa de Alimentación Escolar (PAE) del Ministerio de Educación Nacional, que funciona como una estrategia de alimentación escolar y social financiada con recursos estatales (MEN, 2013) y proyectos de transporte escolar que funcionan de manera intermitente y sirven para brindar acceso al transporte público y garantizar la accesibilidad a la educación (Departamento Nacional de Planeación et al., 2017).

\section{Discusión}

De forma particular en contextos rurales, los proyectos de vida de los jóvenes difieren de los urbanos porque cada integrante en estas comunidades percibe las tareas de agricultura como la principal fuente económica de sustento. Desde edad muy temprana cada persona aprende a realizar el trabajo agrícola que desempeñan sus familiares y encamina los proyectos de vida de sus habitantes hacia estas actividades. Esta información se corrobora en los estudios de García (2017), Guiskin (2019) y Urrutia y Trivelli (2019) quienes plantean que el proyecto vital rural adolescente se ve especialmente intercedido por el trabajo informal en labores agrícolas. De la misma forma, el estudio de Pardo 
(2017) corrobora la importancia que tiene la actividad laboral por encima de la académica para la juventud rural.

En contraste con los hallazgos de la investigación hecha por Garcés-Delgado et al. (2020), fue posible encontrar que los jóvenes en riesgo de exclusión social no están construyendo efectivamente su proyecto de vida debido a la desadaptación a las normas del sistema social por la que transitan. Sin embargo, perciben la realización de sus estudios como un medio para alcanzar la estabilidad económica y la realización personal, a diferencia de los jóvenes rurales que, aunque cuentan con una alta adaptación a las normas comunitarias, comprenden el proceso educativo como un paso innecesario para la realización económica y personal, siendo claro que ambas poblaciones desertan y construyen indebidamente su proyecto vital por causas diferentes. Esto indica que la construcción oportuna de un proyecto de vida se debe abordar contemplando las características específicas de la población. Este es un fenómeno complejo que no tiene una respuesta homogénea o única, sino que requiere de un diagnóstico especializado para darle un abordaje pertinente y funcional, puesto que su configuración dependerá de las particularidades poblacionales.

Se reconoce cómo los jóvenes rurales pasan por una constante contradicción entre lo que anhelan, desean o aspiran y aquello que perciben como su única posibilidad de un proyecto de vida en su entorno, al cual le añaden carga emocional, ansiedad e incertidumbre a la confusión que de por sí ya se presenta en esta etapa vital. Desafortunadamente, son muchos los jóvenes rurales que abandonan sus sueños y se acoplan a las dinámicas sociales, económicas y educativas heredadas de generación en generación, incluso adoptándolas como propias. No se desacredita el trabajo rural, aunque es relevante reconocer que en realidad los adolescentes rurales se plantean construir un proyecto vital de una forma alternativa, pero que al final lo terminan olvidando o destruyendo al percibirlo como inviable (García, 2017; MEN, 2018).

De cara a los hallazgos encontrados, se contempla que la autoestima juega uno de los papeles más representativos como recurso personal, respecto a una elaboración efectiva del proyecto de vida en cuanto a los contextos rurales se refiere. En concordancia con lo argumentado por Castañeda (2011), García (2017) y Sánchez (2019), dentro de los principales elementos a contemplar para elaborar un proyecto vital rural oportunamente está el desarrollo de la autoestima y la motivación, en la medida en que fortalecen la toma de decisiones de forma autónoma y el actuar de manera crítica, así como incentivar el uso del liderazgo en el ámbito familiar, educativo y comunitario. 
Se sostiene que es esencial fortalecer la autoestima para la construcción del proyecto de vida como un paso fundamental que se debe dar, y considerar que existe una alta correlación entre el desarrollo de la autoestima y la construcción efectiva del proyecto vital (Franco, 2017; Gualtero, 2016; Ministerio de Educación [Ecuador], 2018; Vela \& Cáceres, 2019), lo que permitiría incentivar a que los jóvenes rurales desarrollen su inteligencia emocional y tengan un mayor alcance hacia sus metas propuestas. En este orden de ideas y tal como lo expresan Parada et al. (2016), se encontró que una baja autoestima puede relacionarse con un bajo rendimiento académico y una deserción escolar. De forma similar, se contrasta la falta de motivación intrínseca de continuar en un proceso educativo por parte de los adolescentes rurales, a lo largo de diferentes estudios. Al respecto, Pardo (2017) plantea que para los jóvenes rurales no hay un valor agregado en dar continuidad a este proceso y el costo de no poder trabajar es más alto para ellos. Para los hombres jóvenes el poder realizar labores agrícolas es la principal razón para no estudiar, mientras que para las mujeres lo es encargarse de los oficios del hogar.

Lo anterior ayuda a comprender un fenómeno remarcado de comportamiento que se extiende a una parte significativa de la juventud rural entre los 11 y 14 años, que se podría denominar como sesgo del presente o preferencia por la inmediatez. Tal como su nombre lo sugiere, los adolescentes rurales dan prioridad a aquellas actividades que traen un beneficio inmediato a corto plazo, en lugar de invertir tiempo en tareas con una mayor recompensa, pero en un futuro distante. Esto sucede principalmente en aquellos que, desde temprana edad, fueron inmersos en el ámbito laboral agrícola (DNP, 2015; Franco, 2017; Rodríguez-Brito, 2019; Santos-Contreras \& Ortega-Pérez, 2016).

En la presente investigación, y en concordancia con el estudio de Castañeda (2011), se evidencia la necesidad de que los jóvenes rurales identifiquen la importancia de plantear y construir un proyecto de vida a pesar de las dificultades y los obstáculos para afrontar; ello dado que estos objetivos definidos le brindan sentido a permanecer en un proceso educativo, por lo que se considera como un insumo que favorece el mantenimiento de la motivación intrínseca para dar continuidad a este desarrollo educacional, a favor de su proyecto vital y mediante acciones que disminuyan la deserción escolar.

El apoyo familiar se contrasta como uno de los aspectos principales del microsistema, que afecta socioemocional y directamente la toma de decisiones que los jóvenes rurales adoptan de forma continua, incluidos su elección vocacional, el comienzo de una vida laboral temprana o si desertará de su proceso educativo. Estos datos se corroboran en las investigaciones de García (2017) y Herrera y Rivera (2020), y se relacionan con los 
postulados del MEN (2018) cuando plantean que el nivel de educación y el rendimiento escolar de los jóvenes se ven mediados por el grado de participación del círculo familiar en las dinámicas educativas. Los programas diseñados para intervenir esta temática con población joven rural deberían priorizar el análisis de las relaciones existentes entre los familiares, los adolescentes rurales y la institución educativa.

El entorno social con el que esta población está interactuando continuamente es otro de los aspectos más influyentes en la configuración del proyecto de vida, en el cual los pares sociales y los referentes contextuales son los que inciden en la toma de decisiones laborales, profesionales y socioeconómicas, a corto y largo plazo, debido a que es su modelo de referencia en el proceso de socialización e imitación. Es allí donde aprenden a desenvolverse en las distintas circunstancias sociales que provee el contexto (Ibarra et al., 2017 y Ruiz, 2011) y funciona como incentivo para la asistencia escolar mediante la replicación del comportamiento (Rodríguez-Brito, 2019).

En esta investigación en el mesosistema se destacó el actuar de los docentes, los directivos y la orientadora escolar, quienes desempeñaron un rol fundamental en el sentido de que estas prácticas pedagógicas aportan nuevos significados y sedimentan realidades que promueven a que los adolescentes construyan su proyecto de vida desde una mirada autónoma y consciente, a través del desarrollo tanto de saberes como de habilidades, y con el uso de metodologías didácticas y pedagógicas adaptadas a la población. En concordancia con lo que afirman Cárdenas (2010) y Ormaza-Mejía (2019), los educadores son quienes pueden intermediar un vínculo afectivo y de pertenencia por parte de los estudiantes hacia la institución y los procesos educativos que le subyacen, como una dinámica social que resignifica realidades.

El actuar del personal docente en el proyecto de vida rural es determinante en la medida en que impulsa un desarrollo humano integral y formativo en los estudiantes, dotándoles de la capacidad para aumentar la confianza en sí mismos en el afrontamiento de una vida futura (Gualtero, 2016; Ormaza-Mejía, 2019; Valdés-Henao, 2018). En este aspecto, es pertinente que los docentes estén empoderados sobre lo que su actuar representa, puesto que son quienes, más allá de la academia, forman aptitudes o habilidades diferentes a las que el joven rural puede desarrollar con sus familiares o pares; además, son las principales fuentes de información con las que los jóvenes rurales pueden conocer un mundo distinto.

De acuerdo con García (2017), Guiskin (2019), Rodríguez-Brito (2019) y Pardo (2017), y en concordancia con lo encontrado en esta investigación, la inequidad se ve aún más 
remarcada en mujeres rurales y jóvenes, considerando que son excluidas y relegadas a actividades que no se catalogan como el activo productivo de la comunidad; es decir, su papel pasa a ser secundario y enfrentan obstáculos adicionales a causa de las normas sociales establecidas. Por lo anterior, se cuestiona la importancia que les brindarían los habitantes pertenecientes a una comunidad rural a los proyectos de vida construidos por las adolescentes rurales respecto a sus pares masculinos, teniendo en cuenta que tienen un rol definido del cuidado del hogar y los hijos. Por lo tanto, reestructurar el sistema de roles de género culturalmente designados se convierte en un punto clave al que se debe prestar atención en el abordaje del proyecto vital en la ruralidad.

Por otro lado, se identificó la relevancia de que los diferentes actores de la comunidad educativa establezcan relaciones fuertes y efectivas con la finalidad de unir estratégicamente esfuerzos para transformar los proyectos de vida rurales. De acuerdo con lo postulado por el MEN (2018) y Ropero (2016), esta cooperatividad comunitaria permite formar una visión en conjunto entre la institución educativa, los docentes y los padres de familia sobre los procesos que atraviesan los jóvenes rurales y, así, actuar en sinergia, evitando realizar acciones individuales y, por lo tanto, poco eficientes.

Respecto a las políticas públicas relacionadas con la educación rural para jóvenes en Colombia, según los informes del MEN (2018) y Pardo (2017) hay un significativo déficit de programas que actualmente estén implementándose para prevenir la deserción escolar, promover la construcción del proyecto de vida y brindar mayor accesibilidad a la educación de los adolescentes rurales para que desarrollen sus capacidades.

Por tanto, es urgente la implementación y el seguimiento tanto de los proyectos de alimentación, a la infraestructura educativa y al transporte territorial como de la oportunidad de continuar la educación media en el contexto rural. La falta de estos recursos dificulta brindarle a la juventud rural las condiciones educativas óptimas para la permanencia escolar, ya que acrecienta aún más la problemática de deserción escolar y de construir oportunamente un proyecto de vida. Tal como lo sostiene Rodríguez-Brito (2019), han existido problemas en la implementación de programas estatales en lo rural en diferentes zonas del país, a causa de la falta de planeación financiera o el cambio de los medios de transporte como el bus escolar, por lo que la oferta de este servicio no es continua y permanente a lo largo del año escolar, sin mencionar la corrupción en la implementación de programas alimenticios.

En conclusión, la elaboración adecuada de un proyecto de vida en la juventud rural es un proceso en el cual deben empoderarse todos los actores que están involucrados, es 
decir, desde el microsistema hasta el macrosistema. La participación colaborativa e interactiva entre todos los actores (incluidos padres de familia, docentes y directivos institucionales) debe seguir una misma visión y perseguir los mismos objetivos; lo anterior en pro de la oportuna elaboración de un proyecto vital rural y permitir que cada actor aporte a las planeaciones de las acciones para tomar, aunque para ello se requiera inicialmente que cada integrante se empodere del mejoramiento de la calidad de vida a través de la construcción oportuna del proyecto de vida.

En la juventud rural existe una predisposición para considerar otras posibilidades fuera de lo rural, que está latente en los aspectos familiar e individual. La problemática de los adolescentes rurales va ligada al desconocimiento tanto propio como de sus redes de apoyo más cercanas sobre cómo identificar y articular aquellos recursos personales, socioafectivos, comunitarios y materiales en beneficio de su proyecto de vida. Esto explica por qué algunos participantes manifiestan ideas aparentemente contradictorias sobre la disposición que tienen los adolescentes rurales a construir un proyecto de vida diferente al establecido. Para ciertos participantes no hay una disposición observable a ello, pero es claro que existe una realidad alternativa latente esperando a materializarse dentro del mundo simbólico rural.

Se considera que la riqueza de información del presente estudio permitiría comprender otras realidades rurales con características poblacionales similares; sin embargo, existe la necesidad de realizar análisis que incluyan a otras instituciones educativas y comunidades rurales, ya que esta investigación da cuenta de dicha limitación. Además, se resalta la importancia de desarrollar estudios longitudinales del fenómeno y con un número mayor de participantes, en los que se involucre a instituciones gubernamentales.

En el futuro, los resultados de la investigación podrían ser insumo para abordar el proyecto de vida desde el modelo ecológico de Bronfenbrenner, así como construir programas por parte de otros investigadores o entes estatales, para hacer intervenciones con jóvenes rurales de Colombia —o incluso de Latinoamérica - a fin de orientar más oportunamente este proceso vital. De igual manera, se podrá contemplar estos resultados para abordar temáticas en contextos rurales, como igualdad de género, educación sexual, desarrollo psicosocial y dinámicas laborales, sociales y familiares de las comunidades rurales, entre otros. 


\section{Agradecimientos}

Al Centro Educativo Municipal El Campanero y a la Universidad de Nariño por posibilitar espacios investigativos de conocimiento y transformación. A los docentes, padres de familia y a los jóvenes rurales por permitirnos conocer mejor la realidad rural y abrirnos las puertas de su comunidad.

\section{Referencias}

Acevedo, C., Ramírez, M., \& Restrepo, J. (2020). La lúdica como estrategia en la construcción del proyecto de vida para estudiantes del grado 5 de primaria en la Institución Educativa Ciudad Verde [Tesis de especialización, Fundación Universitaria Los Libertadores]. Repositorio Fundación Universitaria Los Libertadores. https://bit.ly/3drcZa8 Ayala, J., \& Carvajal, C. (2016). La relación proyecto de vida, felicidad y rol del docente en estudiantes de la media vocacional. Revista de Investigaciones UCM, 16(28), 102-115. Barbosa-Martínez, R., Ramírez-Aranda, J., Salazar-González, B., Benavides-Torres, R., Champion, J., \& Gallegos-Guajardo, J. (2016). Life project for adolescents: A concept analysis. International Journal of Social Science Studies, 4(5), 31-37. https:// doi.org/10.11114/ijsss.v4i5.1490

Bronfenbrenner, U. (1987). La ecología del desarrollo humano: experimentos en entornos naturales y diseñados. Paidós.

Bronfenbrenner, U. (2005). Making human beings human: Bioecological perspectives on human development. Sage.

Campos, G., \& Martínez, N. (2012). La observación: un método para el estudio de la realidad. Xihmai, 7(13), 45-6o. https://doi.org/10.37646/xihmai.v7i13.202

Cárdenas, S. (2010). Niños y niñas de la calle: coordenadas explicativas del cambio de vida. Revista Latinoamericana de Ciencias Sociales, Niñez y Juventud, 8(2), 1051-1067.

Castañeda, L. (2011). Un plan de vida para jóvenes: ¿qué harás con el resto de tu vida? Panorama. Congreso de la República [Colombia]. (2006). Ley 109o. Diario Oficial 46383. http:// www.secretariasenado.gov.co/senado/basedoc/ley_1090_2006.html

D’Angelo O. (1986). La formación de los proyectos de vida del individuo: una necesidad social. Revista Cubana de Psicología, 3(2), 31-39.

D’Angelo, O. (2000). Proyecto de vida como categoría básica de interpretación de la identidad individual y social. Revista Cubana de Psicología, 17(3), 270-276. 
D’Angelo, O. (2002). El desarrollo profesional creador (DPC) como dimensión del proyecto de vida en el ámbito profesional. Revista Cubana de Psicología, 19(2), 106-114.

Departamento Nacional de Planeación [Colombia]. (2015). El campo colombiano: un camino hacia el bienestar y la paz. Misión para la transformación del campo. https://bit.ly/3DBWWAW

Departamento Nacional de Planeación, Ministerio de Educación Nacional, \& Ministerio de Transporte. (2017). Proyectos tipo: soluciones ágiles para un nuevo país. Departamento Nacional de Planeación. https://bit.ly/zoBxHJB

Díaz-Bravo, L., Torruco-García, U., Martínez-Hernández, M., \& Varela-Ruiz, M. (2013). La entrevista: recurso flexible y dinámico. Investigación en Educación Médica, 2(7), 162-167. https://doi.org/10.1016/S2007-5057(13)72706-6

Franco, P. (2017). Autoestima y proyecto de vida en estudiantes de secundaria de una institución educativa de Villa El Salvador. Acta Psicológica Peruana, 2(1), 33-51.

Fondo Internacional de Desarrollo Agrícola. (2019). Informe sobre el desarrollo rural 2019: crear oportunidades para los jóvenes del medio rural. https://bit.ly/3DojR $3 \mathrm{~B}$

Garcés-Delgado, M., Santana-Vega, L., \& Feliciano-García, L. (2020). Proyectos de vida en adolescentes en riesgo de exclusión social. Revista de Investigación Educativa, 38(1), 149-165. https://doi.org/10.6018/rie.332231

García, K. (2017). Construcción de proyectos de vida alternativos (PVA) en Urabá, Colombia: papel del sistema educativo en contextos vulnerables. Estudios Pedagógicos (Valdivia), 43(3), 153-173. https://doi.org/10.4067/S0718-07052017000300009

Gualtero, M. (2016). Importancia del proyecto de vida, como eje motivacional para el ingreso a estudios superiores, en los estudiantes de los grados $10^{\circ}$ y $11^{\circ}$, de la Institución Educativa Técnica La Chamba, del Guamo-Tolima [Tesis de maestría, Universidad del Tolima]. Repositorio Universidad del Tolima. http://repository.ut.edu.co/handle/oo1/1668 Guiskin, M. (2019). Situación de las juventudes rurales en América Latina y el Caribe. Comisión Económica para América Latina y el Caribe.

Hamui, A., \& Varela, M. (2013). La técnica de grupos focales. Investigación en Educación Médica, 2(5), 55-6o. https://doi.org/10.1016/S2007-5057(13)72683-8

Hernández, R., Fernández, C. \& Baptista, P. (2014). Metodología de la investigación. McGraw-Hill.

Herrera, D., \& Rivera, J. (2020). La educación rural: un desafío para la transición a la educación superior. Revista de Estudios y Experiencias en Educación, 19(41), 87-105. http://doi.org/10.21703/rexe.20201941herrera6

Ibarra, E., Ibarra, T., Martin, E., \& Rincón, M. (2017). Pautas para la construcción del proyecto de vida en jóvenes de $10^{\circ}$ Grado Liceo Nueva Britalia [Tesis de especialización, 
Universidad Distrital Francisco José de Caldas]. Repositorio institucional. http:// hdl.handle.net/11349/5245

Jiménez-Ruiz, I., \& Almansa, P. (2017). La investigación cualitativa ante las prácticas tradicionales perjudiciales. Index de Enfermería, 26(1-2), 5-6.

Klein, A., \& Arantes, V. (2016). Life projects of high school: Young students and school. Educação \& Realidade, 41(11), 135-154. https://doi.org/10.1590/2175-623656117

Lopezosa, C. (2020). Entrevistas semiestructuradas con NVivo: pasos para un análisis cualitativo eficaz. En C. Lopezosa, J. Díaz-Noci, \& L. Codina (Eds.), Methodos: anuario de métodos de investigación en comunicación social (pp. 88-97). Universitat Pompeu Fabra.

Ministerio de Educación [Ecuador]. (2018). Herramientas para orientar la construcción de proyectos de vida de estudiantes. https://bit.ly/3rFfN8y

Ministerio de Educación Nacional [Colombia]. (2013). Lineamientos técnico administrativos del Programa de Alimentación Escolar (PAE). https://bit.ly/2OphLf6

Ministerio de Educación Nacional [Colombia]. (2018). Plan especial de educación rural: hacia el desarrollo rural y la construcción de paz. https://bit.ly/3aL8plm

Ocádiz, A. (2016). La etnometodología: una alternativa relegada de la educación. Ride. Revista Iberoamericana para la Investigación y el Desarrollo Educativo, 6(12), 1-12.

Ormaza-Mejía, P. (2019). Educación: orientación vocacional y profesional, garantía de derechos y construcción de proyectos de vida. Ciencia Unemi, 12(30), 87-102. https:// doi.org/10.29076/issn.2528-7737voli2iss30.2019pp87-102p

Ortega, W., Pozo, F., Vázquez, J., Díaz, E., \& Patiño, A. (2021). Modelo ecológico de Bronferbrenner aplicado a la pedagogía. Modelación matemática para la toma de decisiones bajo incertidumbre: de la lógica difusa a la lógica plitogénica. NSIA Publishing House.

Parada, N., Valbuena, C., \& Ramírez, G. (2016). La autoestima en el proceso educativo: un reto para el docente. Revista Educación y Ciencia, (19), 127-144.

Pardo, R. (2017). Diagnóstico de la juventud rural en Colombia. Grupos de Diálogo Rural, una estrategia de incidencia. Rimisp. https://bit.ly/3tKHlv1

Rodas, F., \& Pacheco, V. (2020). Grupos focales: marco de referencia para su implementación. Innova Research Journal, 5(3), 182-195. https://doi.org/g8n2

Rodríguez-Brito, C. (2019). Estudio de trayectorias y aspiraciones de jóvenes rurales en México. Rimisp. https://bit.ly/2MUL5tn

Ropero, S. (2016). La institucionalidad rural en Colombia: reflexiones para su análisis y fortalecimiento. Mundo Agrario, $17(35)$, e022-e022.

Ruiz, J. (2011). Proyecto de vida: relatos autobiográficos y toma de decisiones. Teoría y Praxis Investigativa, 6(1), 27-35. 
Sánchez, L. (2019). El proyecto de vida como una oportunidad para el fortalecimiento de la identidad individual y social, en jóvenes de grado noveno de la institución educativa tecnoagropecuaria Hernando Borrero Cuadros, Municipio El Cerrito Valle, corregimiento de Tenerife [Tesis de maestría, Universidad Icesi]. Biblioteca digital Icesi. https:// repository.icesi.edu.co/biblioteca_digital/bitstream/10906/85562/1/To1850.pdf

Santos-Contreras, E., \& Ortega-Pérez, D. (2016). Proyecto de vida de los adolescentes del área metropolitana de Cúcuta (zona rural escolar corregimiento de San Faustino). Eco Matemático, 7(1), 112-120. https://doi.org/10.22463/17948231.1022

Schettini, P., \& Cortazzo, I. (2016). Técnicas y estrategias en la investigación cualitativa. Editorial de la Universidad de la Plata.

Schmitt, R., \& Dos Santos, B. (2013). Modelo ecológico del abandono estudiantil en la educación superior: una propuesta metodológica orientada a la construcción de una tesis [Ponencia]. Congresos CLABES. https://revistas.utp.ac.pa/index.php/clabes/article/view/89o

Suárez-Barros, A., Alarcón, Y., \& Reyes, L. (2018). Proyecto de vida: ¿proceso, fin o medio en la terapia psicológica y en la intervención psicosocial? AVFT, Archivos Venezolanos de Farmacología y Terapéutica, 37(5), 505-511.

Urrutia, C., \& Trivelli, C. (2019). Entre la migración y la agricultura: limitadas opciones laborales para los jóvenes rurales en el Perú. RIMISP. https://bit.ly/303 $\mathrm{HP}_{5} \mathrm{H}$

Valdés-Henao, C. (2018). «Deserción» universitaria: entre desvinculaciones institucionales y búsqueda de sentidos de vida. Revista Latinoamericana de Ciencias Sociales, Niñez y Juventud, 16(1), 331-344. https://doi.org/10.1160o/1692715x.16120

Vela, G., \& Cáceres, T. (2019). Educación superior en los proyectos de vida de estudiantes en Arequipa, Perú. Revista de Ciencias Sociales, 25(1), 371-383.

Velasco, M. (2020). Estado del arte: educación rural y saberes tradicionales campesinos [Trabajo de pregrado, Corporación Universitaria Minuto de Dios]. Repositorio Institucional Uniminuto. https://hdl.handle.net/10656/10737 